\title{
La evaluación del desempeño musical y el uso de rúbricas
}

Raúl W. Capistrán Gracia y Juan Pablo Correa Ortega

\section{Introducción}

$\mathrm{E}$ valuar es una tarea permanente en cualquier proceso de enseñanza y de aprendizaje. Su objetivo principal es conocer qué tanto ha aprendido el estudiante, así como la calidad de lo aprendido. Los resultados de la evaluación son de vital consideración; por un lado, proporcionan al maestro información importante para optimizar la labor docente y promover el aprendizaje y el espíritu de superación en los estudiantes; por otro, ofrecen retroalimentación al alumnado sobre sus fortalezas y áreas de oportunidad, lo que le da elementos para mejorar. En ese sentido, Stanley, Brooker y Gilbert (2002: 46) explican:

El propósito esencial de la evaluación educativa es mejorar el aprendizaje. La evaluación proporciona retroalimentación crucial a estudiantes y maestros acerca del progreso en relación al logro de objetivos de enseñanza [...] Un propósito adicional de la evaluación ha surgido recientemente debido al énfasis en la rendición de cuentas en el ámbito de la educación superior.

Dependiendo de las necesidades, las evaluaciones al desempeño musical en una institución de nivel superior pueden servir para aceptar o no a un candidato que desea ingresar a la institución, decidir si un estudiante acredita o no un curso, asignar una calificación, designar un lugar o atril en una orquesta u otorgar un premio en una competencia. Además, para aquellos que ven las evaluaciones como retos y buscan destacar, éstas constituyen en sí mismas una motivación y representan qué tan cerca o lejos se está del ideal artístico.

Este artículo presenta una reflexión acerca de las complejidades inherentes a la evaluación del desempeño de los estudiantes de música en los exámenes, concursos y audiciones; enfatiza la importancia del uso de rúbricas para llevar a cabo una evaluación válida y confiable; y aporta algunas recomendaciones para realizar esta compleja tarea.

Lo que hace compleja la evaluación del desempeño en música es que la apreciación de una ejecución musical, como todo arte, está determinada por aspectos subjetivos. Entre esos aspectos se encuentra lo que el espectador cree sentir al momento de escuchar una ejecución. Si cree haber disfrutado de la ejecución, entonces ésta será evaluada como buena; si la ejecución no fue de su agrado, la juzgará insatisfactoria. No obstante, ¿el espectador es consciente de las razones que le llevaron a disfrutar la ejecución o de lo que no le permitió disfrutarla? Tal vez un espectador común no tendría que ser necesariamente consciente de esto, pero en el caso de un evaluador, especialmente si se trata de un docente, la apreciación sistemática y la consciencia de las razones por las cuales una ejecución es o no aceptable, son tareas esenciales a su labor docente.

Como consecuencia de esta subjetividad, las evaluaciones suelen estar rodeadas de controversia (Navarro, 2013). En ese sentido, Chacón Solís (2012: 1) refiere la siguiente experiencia con 
un estudiante: "En alguna ocasión, uno de ellos me comentó que en un examen final se le había criticado su falta de musicalidad, pero al preguntar qué significaba ser musical y cómo él podía serlo, no obtuvo respuesta".

Por otro lado, el evaluador jamás debe olvidar que está evaluando el desempeño de un ser humano quien, al mostrar sus logros musicales, no sólo está ejecutando notas, sino que está exhibiendo sus aspiraciones y exponiendo su dimensión afectiva. En ese sentido, Prieto Alberola (2001: 329) afirma: "Esto representa una gran responsabilidad para los jurados y tribunales evaluadores, ya que están en juego, a veces, las ilusiones personales y otras su futuro profesional, con las consiguientes consecuencias económicas, sociales y afectivas".

Evaluar una ejecución musical implica medir la calidad de una gran variedad de factores que suceden de manera simultánea en un breve lapso. Afinación, fraseo, articulación, dinámica, agógica, técnica, precisión rítmica, respiración y desenvolvimiento escénico son algunos de esos factores; sin tomar en cuenta la diversidad de parámetros y niveles de desempeño que deben considerarse para medirlos. Saunders y Holahan (1997: 259) explican:

La ejecución musical instrumental representa un acontecer de eventos sonoros a través del tiempo. La compleja naturaleza interactiva de las presentaciones sonoras tonales y rítmicas simultáneas proporcionan un reto para aquellos que intentan escuchar una ejecución musical instrumental con especificidad.

Desde hace algunas décadas, diversos investigadores han tratado de crear e implementar instrumentos de evaluación confiables y válidos que tomen en cuenta los factores y parámetros involucrados en la ejecución musical, los cuales permitan llevar a cabo una evaluación objetiva y justa que refleje el desempeño del estudiante y proporcione información veraz que le posibilite mejorar.

Tradicionalmente, la evaluación al desempeño musical es de tipo holístico, en que el sinodal o jurado emite una sola calificación para medir la calidad total de la ejecución del estudiante. Uno de los primeros intentos por llevar a cabo una evaluación más objetiva fue la evaluación segmentada. En este tipo de evaluación el sinodal otorga un puntaje correspondiente a la calidad de cada dimensión implicada en la ejecución (fraseo, articulaciones, dinámica y agógica, entre otros.). Una variante de este instrumento de evaluación puede incluir descriptores muy básicos como "excelente", "muy bueno", "bueno", "regular", "deficiente", que guían al sinodal sobre la calidad de cada dimensión.

Si bien, es difícil que los jueces o sinodales lleguen a un consenso en cuanto a la calidad de una ejecución, existe la convicción de que hay factores o aspectos para los cuales se tienen estándares comunes de calidad (Abeles, 1973). Partiendo de ese argumento, en la década de 1970 surgieron las "escalas de medición", cuyo patrón se organiza de acuerdo con los factores involucrados en la ejecución, tales como: interpretación, calidad de sonido, continuidad rítmica, afinación, tempo y articulación, etc. Cada factor incluye descriptores completos en la forma de una declaración (ejemplo "El ritmo es irregular"), seguidos de una escala Likert. De esa manera, el evaluador selecciona el nivel o medida en que está de acuerdo con ese descriptor y proporciona una retroalimentación más precisa. Entre los investigadores que han explorado esta forma de evaluación se encuentran Abeles (1973), Bergee (1992), Jones (1986) y Winter (1993).

Aun cuando estas escalas de medición han representado un avance en la calidad de las evaluaciones, también ofrecen algunas desventajas. La principal de ellas radica en que el sinodal o juez se limita a manifestar qué tan de acuerdo está con el descriptor. Como consecuencia, la evaluación resultante no explica por qué una ejecución es excepcional o deficiente.

En la década de 1990, buscando subsanar las áreas de oportunidad que muestran las escalas de medición, investigadores como Azzara (1993), Rutkowski (1990) y Saunders y Holahan (1997), entre otros, desarrollaron un instrumento llamado "escala de medición con criterios específicos de evaluación" (criteria-specific rating scales). En este tipo de instrumento de evaluación, los jueces o sinodales no tienen que decidir si la ejecución musical les gusta o no, o si reúne los estándares de calidad necesarios, sino que deben indicar cuál de los criterios listados dentro de cada una de las dimensiones describe con mayor precisión el nivel de ejecución. Así, su principal ventaja radica en que reduce considerablemente la subjetividad (Saunders y Holahan, 1997). 


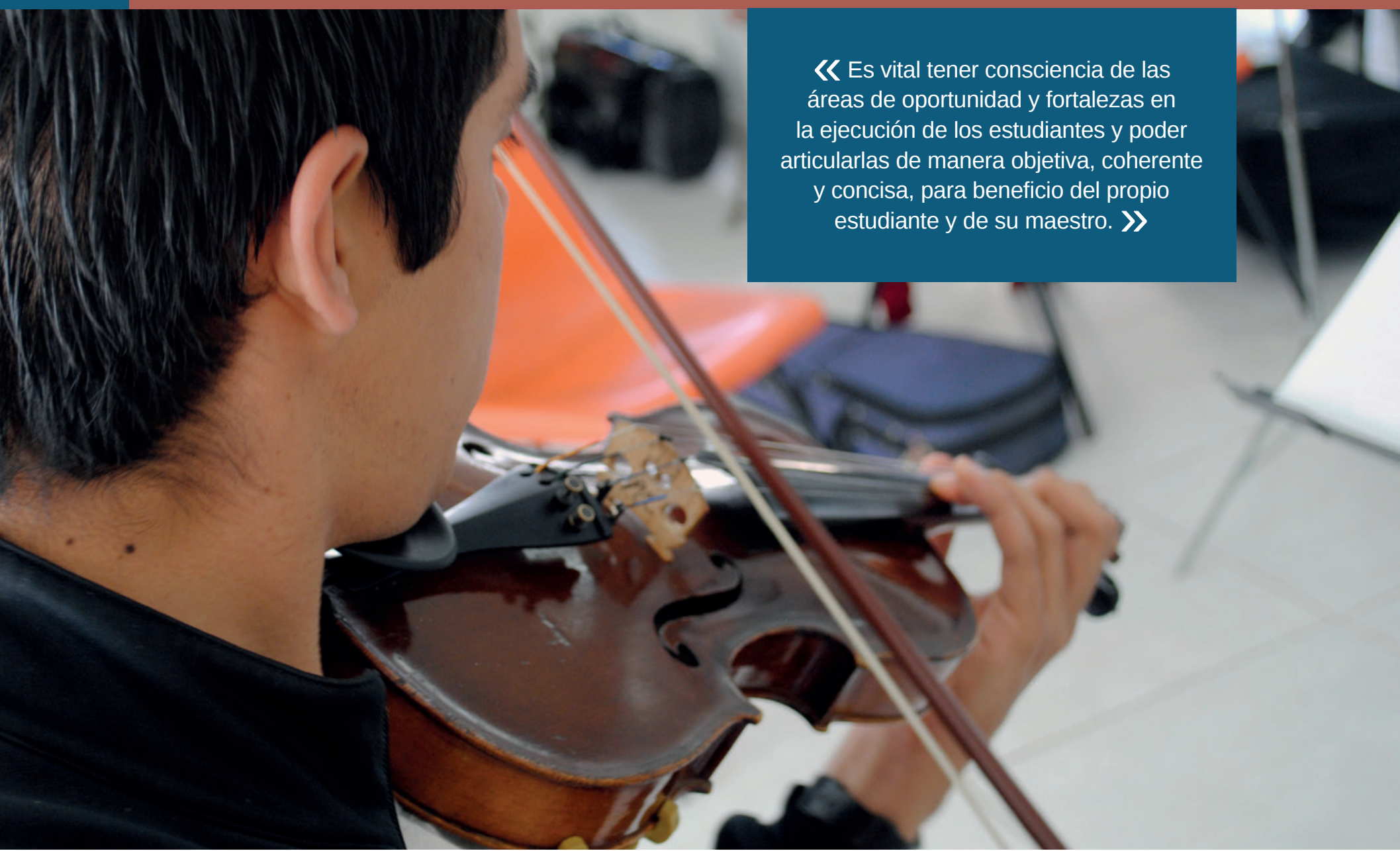

Los instrumentos de evaluación que delimitan las dimensiones a medir y explican los diferentes niveles de desempeño esperados en cada dimensión se conocen como rúbricas. Para la evaluación del desempeño de músicos ejecutantes, o de materias prácticas como el solfeo, donde es usual tener docentes invitados que fungen como co-evaluadores, es fundamental tener un instrumento que facilite la puesta en común de elementos a observar y de criterios de medición de estos elementos dentro del desempeño de cada estudiante. Así, la rúbrica se puede concebir como un resultado de la planeación docente, en tanto se diseña, ex professo, para medir los resultados del aprendizaje que persigue la planeación. Al respecto, Correa y Pérez-Martínez (2015), a través de entrevistas a docentes del Departamento de Música de la Universidad Autónoma de Aguascalientes, encontraron que algunos de los profesores no sólo consideran útiles las rúbricas para evaluar, sino también para la planeación de la enseñanza en sus cursos de instrumento principal.
Por otro lado, es importante no perder de vista que la evaluación, como proceso humano, es, por naturaleza, subjetiva, y esto, como afirma Mills (1991), debe celebrarse, pues allí estriba la diversidad de la vida; sin embargo, también es vital proporcionar la mejor retroalimentación posible a estudiantes y maestros después de una presentación, pues de esto depende el mejoramiento del proceso de enseñanza y de aprendizaje, así como la superación del estudiante. Ciertamente, después de terminar una presentación uno no dice "las notas fueron correctas, el ritmo era correcto, el fraseo era coherente, etc., por lo tanto, disfruté de la ejecución" (Mills, 1991: 175); sin embargo, es vital tener consciencia de las áreas de oportunidad y fortalezas en la ejecución de los estudiantes y poder articularlas de manera objetiva, coherente y concisa, para beneficio del propio estudiante y de su maestro, algo que desgraciadamente muchas veces no ocurre (Alberola, 2001). Después de todo, si enseñamos a nuestros estudiantes a entender y do- 
minar "los diferentes elementos de la música, para luego integrarlos en una forma artística coherente, [los maestros] también deberíamos evaluar estos elementos en la ejecución musical [de nuestros estudiantes] y emitir un juicio integrado" (Winter, 1993: 37).

\section{Conclusión}

Existen diversas alternativas, como el portafolio de evidencias, la autoevaluación y la evaluación por pares que pueden contribuir enormemente a llevar a cabo una evaluación válida y confiable, y que pueden proporcionar una retroalimentación efectiva a estudiantes y maestros sobre el desempeño musical y el proceso de aprendizaje. No obstante, por el momento, las rúbricas son el instrumento oficial mejor adaptado a la evaluación de los exámenes de ejecución y audiciones de puestas en escena. Si bien, tienen áreas de oportunidad, un diseño congruente con los objetivos de aprendizaje o selección de aspirantes, y un uso ético de las mismas, permitirá llevar a cabo procesos de evaluación más neutrales, transparentes y que informen de manera eficiente los procesos de enseñanza y de aprendizaje.

Fuentes de consulta

Abeles, H. (1973). Development and validation of a clarinet performance adjudication scale. Journal of Research in Music Education, 21(3), 246-255. Recuperado de http://www. jstor.org/stable/3345094.

Azzara, C. (1993). Audiation-based improvisation techniques and elementary instrumental students' music achievement. Journal of Research in Music Education, 41(4), 328342.

Bergee, M. (1992). A scale assessing music student teachers' rehearsal effectiveness. Journal of Research in Music Education, 40, 5-13.

Chacón-Solís, L. (2012). ¿Qué significa "evaluar" en música? Revista Electrónica Complutense de Investigación en Educación Musical, 9, 1-25. Recuperado de http://dx.doi. org/10.5209/rev_RECI.2012.v9.42805 ISSN 1698-7454.

Correa, J. y Pérez-Martínez, M. (2015). Implementación de rúbricas de evaluación en el Departamento de Música de la UAA. En R. W. Capistrán, Educación musical a nivel superior, 44-53. Aguascalientes: UAA.

Jones, H. Jr. (1986). An Application of the Facetfactorial Approach to Scale Construction in the Development of a Rating Scale for High School Vocal Solo Performance (adjudication, Evaluation, Voice) (Order No. 8613727). Disponible en ProQuest Dissertations \& Theses Global. (303512065). Re- cuperado de https://search.proquest.com/do cview/303512065?accountid=151090.

Mills, J. (1991). Assessing musical performance musically. Educational Studies, 17(2), 173181.

Navarro, J. (2013). La evaluación musical en una escuela universitaria mexicana: opiniones del alumnado. Revista Electrónica de Música en la Educación, 32, 19-52.

Prieto-Alberola, R. (2001). La evaluación de las actividades musicales. Contextos educativos, 4, 329-340.

Rutkowski, J. (1990). The measurement and evaluation of children's singing voice development. The Quarterly, 1(1-2), 81-95.

Saunders, T. C. y Holahan, J. M. (1997). Criteria-specific rating scales in the evaluation of high school instrumental performance. Journal of Research in Music Education, 45, 259-272. doi: 10.2307/3345585.

Stanley, M., Brooker R. y Gilbert, R. (2002). Examiner perceptions of using criteria in music performance assessment. Research Studies in Music Education, 18, 46. doi: 10.1177/1321103X020180010601.

Winter, N. Music. (1993). Performance assessment: A study of the effects of training and experience on the criteria used by music examiners. International Journal of Music Education, 22(1), 34-39. 